\title{
Autofluorescence For Rapid Visualization of Plant Anatomy Among Diverse Taxa
}

\author{
Timothy Pegg, Daniel Gladish and Robert Baker
}

Miami University, Oxford, Ohio, United States

Examining the genetic architecture of relationships between internal plant anatomic structures, ecophysiological function, and organismal morphology promises to reveal new avenues for plant breeding and modeling complex ecological/evolutionary dynamics in natural systems (He et al., 2017; Baker et al., 2017). However, high-throughput methods for assessing internal anatomy remain elusive, precluding the widespread inclusion of internal anatomy in many modern 'omics level studies. Internal anatomy remains difficult to assess for several reasons. First, most techniques involve destructively sampling from the plant. Live-imaging techniques exist (Fang \& Spector, 2010; Mathers et al., 2018); however, these can be cost prohibitive, are often low throughput, and require that plants are grown in artificial conditions. Second, although it is possible to reconstruct internal anatomy at fairly high resolution after destructive sampling (Miki et al., 2020), this method is time-consuming due to lengthy fixation, embedding, sectioning, and staining steps. In this project, we present minimal sample preparation and microscopy protocols that utilize inherent autofluorescence in plant internal structures for markedly increased throughput in cellular and ultrastructural visualization.

In our experimental design we selected eleven species to demonstrate the applicability of our protocol across a phylogenetically broad range of plant taxa (Fig. 1): Brassicaceae Arabidopsis thaliana (L.), Poaceae Zea mays (L.), Fabaceae Glycine max (L.), Brassicaceae Brassica rapa (L.), Pinaceae Pinus strobus (L.), Volvocaceae Volvox sp (L.), Ulvaceae Ulva sp. (L.), Marchantiaceae Marchantia sp. (L.), Polyphysaceae Acetabularia sp., Coleochaetaceae Coleochaete sp., and Selaginellaceae Selaginella sp. Five samples from each species were collected and placed separately into the following solutions: $2 \%$ paraformaldehyde in $0.1 \mathrm{M}$ sodium cacodylate

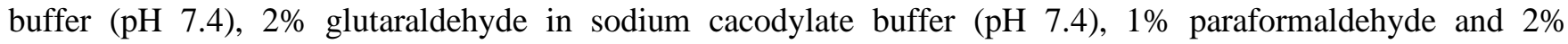
glutaraldehyde in 0.1M sodium cacodylate buffer ( $\mathrm{pH} 7.4$ ), Formalin-acid-alcohol (Ruzin, 1999), or 70\% ethanol. Samples were then mounted in glycerol and imaged on an Olympus FV500 Confocal Laser Scanning system. Three channels were recorded for each sample, representing aldehyde-induced fluorescence (Alexa Fluor 405 and FITC and filters) and chlorophyll autofluorescence (Texas Red filter). Enhancement of micrographs was performed using FIJI imaging software (Schindelin et al., 2012) to adjust contrast/brightness and assign green, blue and red LUTs for image channels corresponding to fluorescence in the $405 \mathrm{~nm}, 433 \mathrm{~nm}$ and $647 \mathrm{~nm}$ ranges, respectively. Image channels were then overlaid to form a composite image for each sample.

Trends in fluorescence were examined among treatments and species with the goal of clearly identifying cellular structures. Samples preserved with PFA, Glut and PFA/Glut demonstrated notable fluorescence of cell walls across all taxa (Fig. 2). Plants belonging to the tracheophytes showed consistent fluorescence with leaf tissue from Zea mays (Fig. 2, F-H) representing excellent examples of clear cell wall, xylem, phloem, nuclei, and chloroplast definition. Samples preserved with FAA demonstrated poor/mottled chlorophyll fluorescence patterns in taxa belonging to the Chlorophytes (Ulva, Acetabularia, and Volvox) (Fig. 2D) and Charophytes (Coleochaete and Chara). By contrast, taxa belonging to the Tracheophytes displayed adequate definition of cell walls, but similar loss of chlorophyll fluorescence (Fig. 2I). Zea mays, Glycine max, Marchantia and several green algae samples demonstrated similar patterns of fluorescence when fixed with $70 \%$ ethanol, suggesting native cell wall autofluorescence emission wavelengths partially overlap with those of aldehyde-induced fluorescence (Fig. 2J). By comparison Volvox and most of the Tracheophytes show little cell wall fluorescence in samples preserved with $70 \%$ ethanol (Fig. 1E, J).

Trends in taxa structural preservation due to fixative treatments were also evaluated. Tracheophyte samples demonstrated little change in structural quality across treatments, with clearly delineated regions of mesophyll, epidermal and vascular tissues in many samples (Fig. 2F-J). However, fixatives containing alcohol caused substantial damage in tissues belonging to aquatic plants, or those with thinner cell walls. For example, FAA fixation 
resulted in minimal damage to tracheophyte samples, but caused cell shrinkage and lysis in Chlorophycean and Streptophycean green algae and Marchantia. FAA-induced cell damage was prominent in Chara, Ulva, Volvox and Acetabularia. Poor structural preservation of FAA-treated samples may be the result of rapid infiltration with ethanol, since similar cell damage was also observed when these samples were preserved with $70 \%$ ethanol. In addition, ethanol content leached chlorophyll from numerous samples, resulting in damage to chloroplasts (Fig 2E, J).

Historically, plant cell morphology research relied on time consuming techniques such as histological staining, immunolabeling, electron microscopy preparations, or limitations in bright field microscopy resolution. Integrating internal anatomy with modern molecular and morphological datasets requires quickly/easily generating anatomical information (Li \& Chen, 2014). Here, we demonstrate how simple fixation protocols and confocal light microscopy may be used to rapidly observe aldehyde-induced fluorescence to gather cellular structure data from multiple plant phyla. Our protocols serve as a guide for proper plant fixative selection for a given taxa and an illustration of possible preservation artifacts. Using thee protocols to increase throughput for anatomical studies may improve our understanding of plant evolutionary biology and the precision of plant breeding programs.

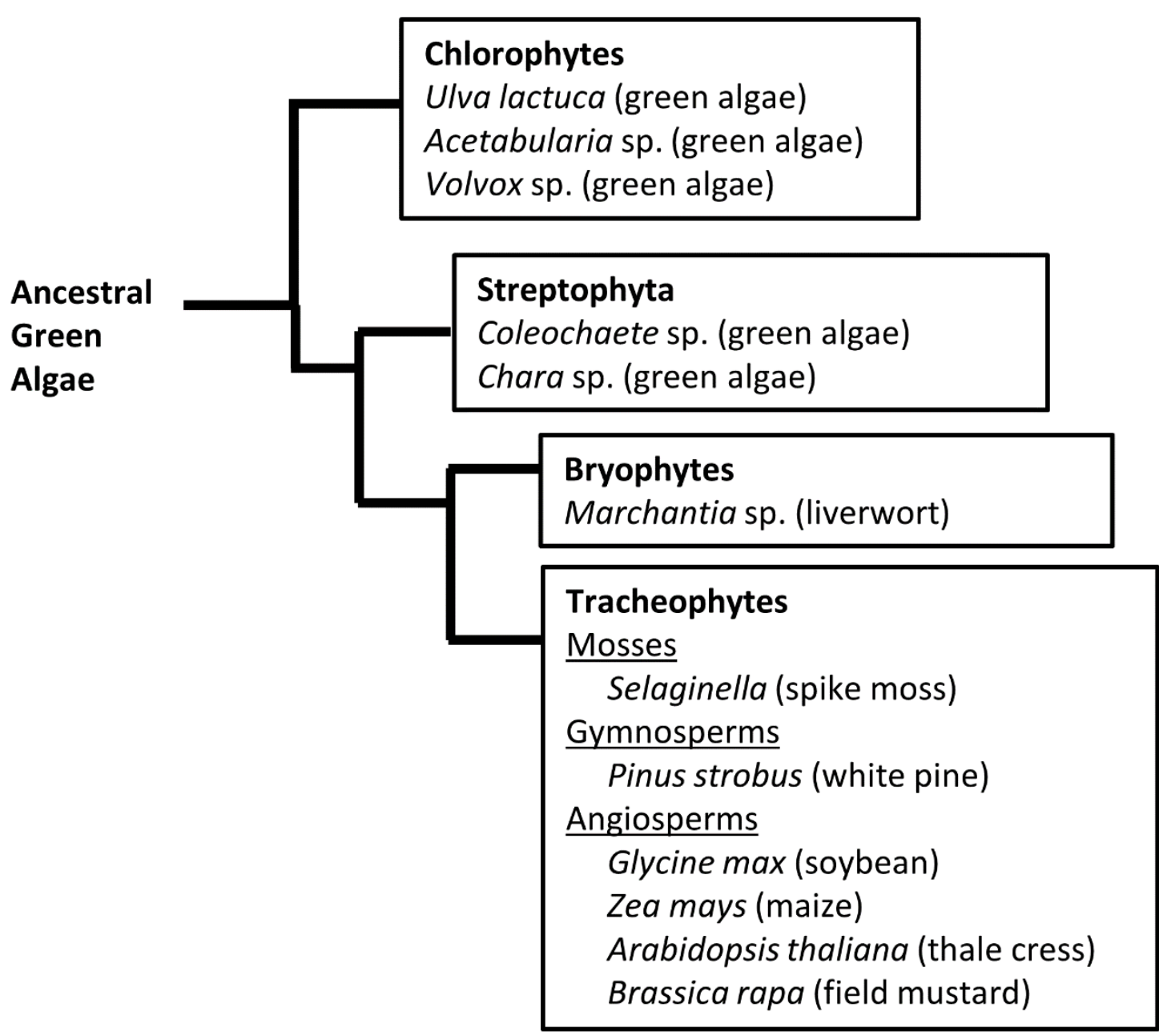

Figure 1. Phylogenetic tree representing evolutionary relationships between twelve species utilized in this protocol, adapted from Leebens-Mack et al., 2019. 

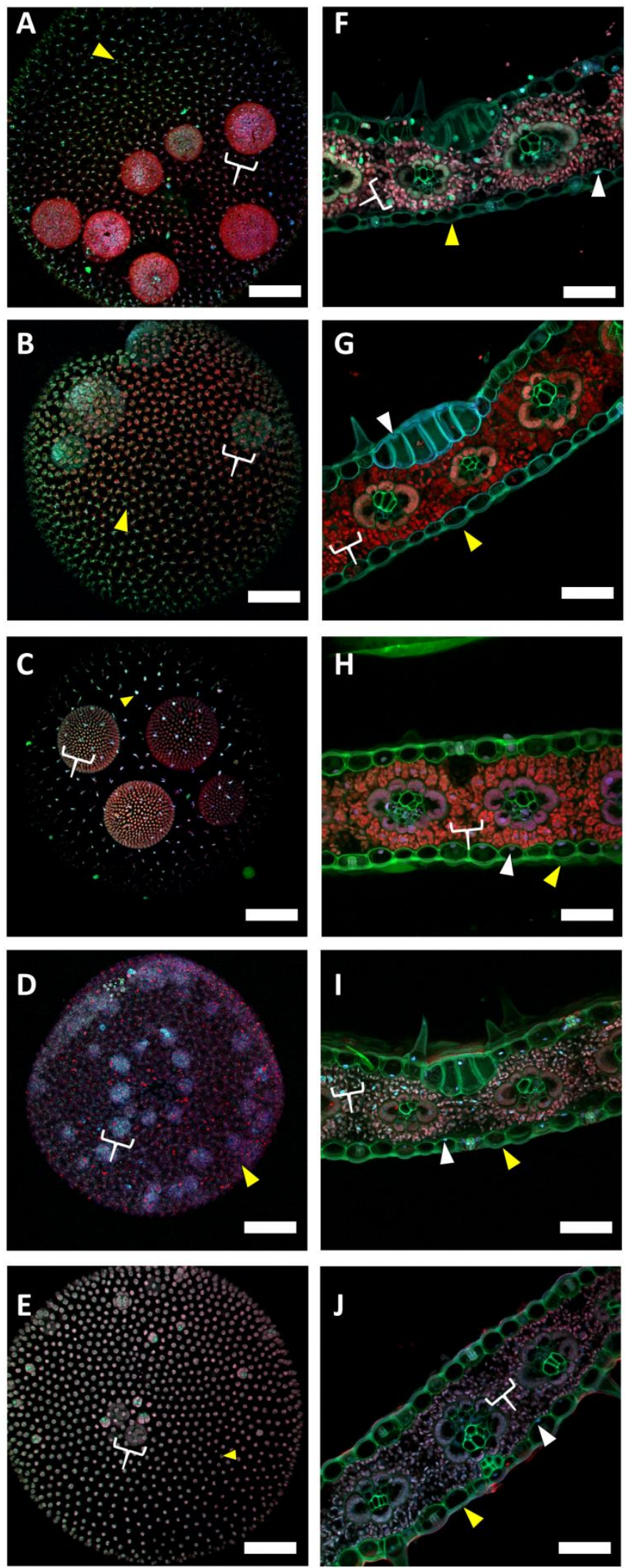

Figure 2. Images of Volvox colonies (A-E) and Zea mays leaf cross-sections (F-J). Each image demonstrates fluorescence of tissues subjected to different fixation treatments. Fixatives represented are 
(A, F) $1 \%$ Paraformaldehyde and 2\% glutaraldehyde, (B, G) 2\% Paraformaldehyde, (C, H) 2\% Glutaraldehyde, (D, I) Formalin-Acetic Acid (FAA), and (E, J) 70\% ethanol treatment. False-color represents fluorescence through Alexa Fluor 405 (green), FITC (blue) and Texas Red (red) bandpass filters. Cell walls, nuclei and chlorophyll are indicated by yellow wedges, white wedges, and white brackets, respectively. Scales bars $=50 \mu \mathrm{m}$.

References

Baker, R. L., Yarkhunova, Y., Vidal, K., Ewers, B. E. \& Weinig, C. (2017). Polyploidy and the relationship between leaf structure and function: implications for correlated evolution of anatomy, morphology, and physiology in Brassica. BMC Plant Biology 17, 3. https://doi.org/10.1186/s12870-0160957-3.

FAnG, Y. \& Spector, D. L. (2010). Live Cell Imaging of Plants. Cold Spring Harbor Protocols 2010, pdb.top68. http://cshprotocols.cshlp.org/content/2010/2/pdb.top68.abstract.

He, W., Adachi, S., Sage, R. F., Ookawa, T. \& Hirasawa, T. (2017). Leaf photosynthetic rate and mesophyll cell anatomy changes during ontogenesis in backcrossed indica $\times$ japonica rice inbred lines. Photosynthesis Research 134, 27-38. https://doi.org/10.1007/s11120-017-0403-x.

LI, Y. \& CHEN, L. (2014). Big biological data: challenges and opportunities. Genomics, proteomics \& bioinformatics 12, 187-189. https://pubmed.ncbi.nlm.nih.gov/25462151.

Mathers, A. W., HePworth, C., Baillie, A. L., Sloan, J., Jones, H., Lundgren, M., Fleming, A. J., MoOneY, S. J. \& STURROCK, C. J. (2018). Investigating the microstructure of plant leaves in 3D with labbased X-ray computed tomography. Plant Methods 14, 99. https://doi.org/10.1186/s13007-018-0367-7.

Miki, Y., SAITO, S., NiKI, T. \& GLADISH, D. K. (2020). Three-dimensional digital image construction of metaxylem vessels in root tips of Zea mays subsp. mexicana from thin transverse sections. Applications in Plant Sciences 8, e11347. https://doi.org/10.1002/aps3.11347.

RuZIN, S. E. (1999). Plant microtechnique and microscopy. Oxford University Press New York.

Schindelin, J., Arganda-CARreras, I., Frise, E., Kaynig, V., LOngair, M., PiETzSCH, T., Preibisch, S., Rueden, C., SAAlfeld, S., Schmid, B., Tinevez, J.-Y., White, D. J., HARTEnStein, V., Eliceiri, K., TOMANCAK, P. \& CARDONA, A. (2012). Fiji: an open-source platform for biological-image analysis. Nature Methods 9, 676-682. https://doi.org/10.1038/nmeth.2019. 